\section{A CHECKLIST OF LAND AND FRESHWATER MOLLUSCA OF MAHARASHTRA STATE}

\section{S.G. Patil and S.S. Talmale}

Zoological Survey of India, Western Regional Station, Sector 29, Vidyanagar, Rawet Road, P.C.N.T. Post, Pune, Maharashtra 411044, India

Molluscs occur in various habitats and are divided into freshwater, marine and terrestrial forms. The freshwater molluscs play a significant role in aquatic ecosystems, and some of them are edible. Species like Bellamya bengalensis, Pila globosa and Lamellidens marginalis are proven food for many aquatic animals and man; some such as Lamellidens marginalis and Lamellidens corrianus have also been used to produce pearls in some parts of India (Subba Rao \& Dey, 1989). Shells of some of molluscs are used for making buttons and in manufacture of poultry food. Some of them are also intermediate hosts for parasites, like Pila globosa, Lymnaea etc. (Subba Rao, 1989).

Maharashtra state with three biogeographic zones (West Coast, Western Ghats \& Deccan plataeu) is located between $15^{\circ} 8^{\prime}$ $22^{\circ} 1^{\prime} \mathrm{N}$ and $72^{\circ} 6^{\prime}-80^{\circ} 9^{\prime} \mathrm{E}$ with a total area over $3,07,690 \mathrm{~km}^{2}$ and a coast line of $720 \mathrm{~km}$ in the west. The molluscan fauna of this state has not been studied thoroughly and the available information on the freshwater and land molluscs is scattered. Perusal of literature shows that a few workers like Blanford (1863, 1870, 1880), Preston (1915), Annandale (1919), Annandale and Prashad (1919), Hora (1925), Tonapi and Mulherkar (1963), Tonapi (1971), Subba Rao and Mitra (1979), Subba Rao (1989), Surya Rao et al. (2002), Patil (2003), and Patil and Talmale (2003) have made contributions. However, except for Subba Rao and Mitra (1979) there is no updated information available on this group in Maharashtra.

This paper lists altogether 142 species of molluscs with all forms and varieties belonging to 48 genera, under 23 families. This checklist of land and freshwater mollusca of Maharashtra state is based on past and present records, and from recent surveys made in this area by many parties of Western Regional Station, Zoological Survey of India, Pune. There is still a need for detailed study of the fauna of Western Ghats in Maharashtra. Information on ecology and systematics of land molluscs such as slugs and amphibian molluscs is grossly inadequate. Further, extensive surveys of this area will undoubtedly reveal the existence of many more species than what is known today.

\footnotetext{
REFERENCES

Annandale, N. (1919). The fauna of certain small streams in Bombay Presidency. Records of the Indian Museum 16: 117-120.

Annandale, N. and B. Prashad (1919). Some freshwater Mollusca from the Bombay Precidency. Records of the Indian Museum 16: 139$152+4,5$ pls.

Blanford, W.R. (1863). Descriptions of Cremnocochus syhadrensis and Lithotis rupicola two new generic forms of Mollusca inhabiting
} cliffs in the Western Ghats of India. Annals and Magazine of Natural
History (3)12: 184-187+4pls

Blanford, W.T. (1870). Contributions to Indian Malacology, No.XI Descriptions of new species of Paludomus, Cremnoconchus, Cyclostoma and of Helicidae from various parts of India. Journal of the Asiatic Society of Bengal 39(2): 9-25+3pls.

Blanford, W.T. (1880). Contributions to Indian Malacology No.XII, Descriptions of new land and freshwater shells from southern and western India, Burmah, the Andaman Islands. Journal of the Asiatic Society of Bengal 49(2): 181-222+I,IIpls.

Blanford, W.T. and H.H.G. Austen (1908). The Fauna of British India, including Ceylon and Burma, Mollusca I: Testacellidae and Zonitidae. Taylor \& Francis, London, 311pp.

Gude, G.K. (1914). The Fauna of British India including Ceylon and Burma, Mollusca II: Troknomorphidae and Jancllidae. Taylor and Francis, London, 520pp.

Gude, G,K. (1921). The Fauna of British India including Ceylon and Burma, Mollusca III: Land operculates. Taylor \& Francis, London, $336 \mathrm{pp}$.

Hora, S.L. (1926). On some interesting features of the Western Ghats, Journal of the Bombay Natural History Society 31: 447-449.

Patil S.G. (2003). Occurrence of freshwater Bivalves (Bivalvia: Unionidae) in Pusad, Yavatmal district, Maharashtra. Zoos' Print Journal 18(9): 1195

Patil, S.G. (in press). Freshwater Mollusca of Melghat Tiger Project Maharashtra State. Fauna of Conservation area series, Zoological Survey of India Publication.

Patil, S.G. and S.S. Talmale (2003). Occurrence of a pestiferous land Mollusca from Maharashtra on new host plants. Bionotes 5(3): 71

Preston, H.B. (1915). Fauna of British India including Ceylon and Burma, Mollusca: Freshwater Gastropoda and Pelecypoda. London, xix $+244 p p$.

Rao, H.S. (1925). On certain succineid Molluscs from the Western Ghats, Bombay Presidency. Records of the Indian Musuem 27: 385 400 .

Rao, V.T. and F. Ramadoss (1953). Damage to vegetable crops by slugs observed in the Krishna District and the experiments on their control. Journal of the Zoological Society of India 5: 211-216.

Subba Rao, N.V. (1989). Handbook of Freshwater Molluscs of India. Zoological Survey of India, Calcutta, 289pp.

Subba Rao, N.V. and A. Dey (1989). Freshwater Molluscs in Aquaculture, pp. 225-232. In: Handbook of Freshwater Molluscs of India. Zoological Survey of India, Calcutta, 289pp.

Subba Rao, N.V. and S.C. Mitra (1975). On collections of Mollusca from Poona and adjacent districts. Newsletter of the Zoological Survey of India 1(4): 77-79.

Subba Rao, N.V. and S.C. Mitra (1979). On land and freshwater Molluscs of Pune district, Maharashtra. Records of the Zoological Survey of India 75: 1-37.

Surya Rao, K.V., S.C. Mitra and S. Maitra (2002). Mollusca of Ujani Wetland, pp. 110-115. Wetland Ecosystem Series 2: Fauna of Ujani. Zoological Survey of India, Kolkata.

Tonapi, G.T. and L. Mulherkar (1963). On the freshwater molluscs of Poona. Journal of the Bombay Natural History Society 60(1): 104$120+\mathrm{i}-\mathrm{v}+\mathrm{Map}$.

Tonapi, G.T. (1971). Studies on the freshwater and amphibious Mollusca of Poona with notes on their distribution - Part II. Journal of the Bombay Natural History Society 68(1): 115-126.

\section{ACKNOWLEDGEMENTS}

The authors are thankful to Dr. J.R.B. Alfred, Director,Zoological Survey of India, Kolkata and to Dr. Anil Mahabal, Officer-in-Charge, Zoological Survey of India, Western Regional Station, Pune for facilities. Thanks are also due to Dr. M.S.Pradhan, Scientist-D, for going through the manuscript. 


\section{Table 1. Checklist of land and freshwater molluscs of Maharashtra}

Phylum: Mollusca

Class: Gastropoda

Order: Mesogastropoda

Family: Cyclophoridae

Cyathopoma deccanense Blanford

Cyclophorus (Annularia) aurantiacus (Schumacher)

Cyclophorus (Glossostylus) indicus (Deshayes)

Cyclophorus (Litostylus) involvulus (Muller)

Cyclophorus (Glossostylus) stenomphalus Pfeiffer

\section{Family: Diplommatinidae}

Nicida liricincta Blanford

Opisthostoma fairbanki Blanford

Family: Pomatiasidae

Cyclotopsis semistriata (Sowerby)

\section{Family: Viviparidae}

Subfamily: Bellamyinae

Bellamya bengalensis form annandalai (Kobelt)

Bellamya bengalensis form bengalensis (Lamarck)

Bellamya bengalensis form doliaris (Gould)

Bellamya bengalensis form eburnea (Annandale)

Bellamya bengalensis form incrassatus Annandale

Bellamya bengalensis form mandiensis Kobelt

Bellamya dissimilis (Muller)

Family: Pilidae

Pila globosa (Swainson)

Pila virens (Lamarck)

Turbinicola saxea (Reeve)

Family: Hydrobiidae

Subfamily: Bithyninae

Digoniostoma cerameopoma (Benson)

Digoniostoma pulchella (Benson)

Gabbia orcula Frauenfeld

Gabbia orcula var. producta (Nevill)

Gabbia stenothyroides (Dohrn)

Sataria evezardi (Blanford)

\section{Family: Thiaridae}

Subfamily: Thiariinae

Thiara (Tarebia) lineata (Gray)

Thiara (Melanoides) pyramis (Hutton)

Thiara (Melanoides) pyramis var. leopardina

Annandale \& Prashad

Thiara (Melanoides) pyramis var. puteicola

Annandale \& Prashad

Thiara (Melanoides) riqueti (Grateloup)

Thiara (Thiara) scabra (Muller)

Thiara (Thiara) scabra var. elegans (Benson)

Thiara (Melanoides) tuberculatus (Muller)

\section{Subfamily: Melanatrinae}

Sulcospira (Sulcospira) hugely var. compacta Nevill

Subfamily: Paludominae

Paludomus (Paludomus) obesus (Philippi)

Paludomus (Stomatodon) stomatodon Benson

Paludomus tanschaurica var. malabarica Nevill

Subfamily: Melanopsinae

Faunus ater (Linnaeus)

Family: Littorinidae

Cremnoconchus (Cremnoconchus) syhadrensis

(Blanford)

Cremnoconchus (Lissoconchus) conicus (Blanford)

Cremnoconchus conicus var. edecollata Nevill

Cremnoconchus (Lissoconchus) carinatus (Layard)

Order: Basommatophora

Family: Lymnaeidae

Lymnaea (Pseudosuccinea) acuminata (Lamarck)

Lymnaea (Pseudosuccinea) acuminata f. gracilior

Martens

Lymnaea (Pseudosuccinea) acuminata var. nana

Annandale

Lymnaea (Pseudosuccinea) acuminata f. patula

Troschel
Lymnaea (Pseudosuccinea) acuminata f. rufescens

Gray

Lymnaea (Pseudosuccinea) acuminata ssp. tonapii

Ray \& Roy Choudhury

Lymnaea (Pseudosuccinea) acuminata f. typica

Lamarck

Lymnaea (Radix) auricularia (Linnaeus)

Lymnaea (Pseudosuccinea) brevissima (Annandale

\& Rao)

Lymnaea (Pseudosuccinea) luteola (Lamarck)

Lymnaea (Pseudosuccinea) luteola f. australis

Annandale \& Rao

Lymnaea (Pseudosuccinea) luteola f. impura

Troschel

Lymnaea (Pseudosuccinea) luteola f. succinea

Deshayes

Lymnaea (Pseudosuccinea) luteola f. typica

Lamarck

Lymnaea pinguis Dohrn

Family: Planorbidae

Subfamily: Buliniae

Indoplanorbis exustus (Deshayes)

\section{Subfamily: Planorbinae}

Gyraulus convexiusculus (Hutton)

Gyraulus labiatus (Benson)

Gyraulus rotula (Benson)

Order: Systellommatophora

Family: Veronicellidae

Laevicaulis alte (Ferussac)

Sarasinula plebeja (Fischer)

Semperula birmanica (Theobald)

Semperula maculata (Templeton)

Order: Stylommatophora

Family: Succineidae

Indosuccinea khandalla Rao

Lithotis rupicola Blanford

Lithotis tumida Blanford

Succinea bensoni Pfeiffor

Succinea collina Hanley \& Theobald

Succinea raoi Subba Rao and Mitra

Succinea tornadri Rao

\section{Family: Cerastuidae}

Subfamily: Napaeinae

Cerastus abyssinicus (Pfeiffer)

Cerastus densus (Pfeiffer)

Cerastus distans (Pfeiffer)

Cerastus jerdoni (Reeve)

Cerastus jerdoni var. redfieldi (Pfeiffer)

Cerastus moussonianus (Petit)

Rachis bengalensis Lamarck

Rachis praetermissus Blanford

Rachis punctatus (Anton)

Family: Subulinidae

Subfamily: Subulininae

Glessula arthuri (Benson)

Glessula brevis (Pfeiffer)

Glessula ceylanica (Pfeiffer)

Glessula chessoni (Benson)

Glessula dikrangense Godwin-Austen

Glessula gemma (Reeve)

Glessula hebes (Blanford)

Glessula illustris var. tumida Godwin-Austen

Glessula lyrata Blanford

Glessula notigena (Benson)

Glessula pulla Blanford

Glessula rugata Blanford

Glessula singhurensis Blanford

Glessula tornensis Blanford

Family: Opeatinae

Opeas gracile (Hutton)

Subfamily: Rumininae

Zootecus chion (Pfeiffer)
Zootecus insularis (Ehrenberg)

Family: Ariophantidae

Subfamily: Ariophantinae

Ariophanta bajadera (Pfeiffer)

Ariophanta laevipes (Muller)

Cryptozona (Nilgiria) semirugata (Beck)

Cryptozona (Xestina) belangeri var. bombayana

(Pfeiffer)

Cryptozona (Xestina) bistrialis (Beck)

Euplecta subdecussata Pfeiffer

Subfamily: Kaliellinae

Kaliella barrackporensis Pfeiffer

Kaliella bullula (Hutton)

Subfamily: Macrochlamydinae

Macrochlamys indica (Godwin \& Austen)

Macrochlamys infaustra Blanford

Macrochlamys pedina (Benson)

Macrochlamys petasus (Benson)

Macrochlamys petrosa (Hutton)

Macrochlamys tenuicula $(\mathrm{H}$. Adams)

Subfamily: Girasiinae

Mariaella dussumieri Gray

Subfamily: Durgellinae

Sitala denselirata Preston

Subfamily: Helicarioninae

Eyrychlamys platychamys Blanford

Pseudaustenia atra Godwin-Austen

Family: Trochomorphidae

Trochomorpha (Sivela) billiana (Morch)

Family: Pleurodontidae

Chloritis leithi Gude

Chloritis propinqua (Pfeiffer)

Planispira (Trachia) crassicostata (Benson)

Planispira footei Stoliczka

Planispira proxima (Ferussac)

Family: Fruticicoloidae

Eulota scalpturita (Benson)

Family: Vallonidae

Pupisoma evezardi (Blanford)

Family: Streptaxidae

Streptaxis scalptus Blanford

Class: Bivalvia

Order: Eulamellibranchiata

Family: Unionidae

Subfamily: Unioninae

Lamellidens consobrinus (Lea)

Lamellidens corrianus (Lea)

amellidens lamellatus (Lea)

Lamellidens marginalis (Lamarck)

Lamellidens marginalis var. cylindrica (Hanley \&

Theobald)

Subfamily: Parreysiinae

Parreysia (Radiatula) caerulea Lea

Parreysia (Parreysia) corrugata Muller

Parreysia (Parreysia) corrugata (Muller) sub sp. laevirostris (Benson)

Parreysia corrugata var. nagpoorensis (Lea)

Parreysia (Parreysia) cylindrica Annandale \& Prashad

Parreysia favidens (Benson)

Parreysia favidens var. marcens (Benson)

Parreysia (Radiatula) khadakvaslaensis (Ray)

Parreysia rajahensis (Lea)

Family: Corbiculidae

Corbicula krishnaea Ray

Corbicula peninsularis Prashad

Corbicula regularis Prime

Corbicula striatella Deshayes 